\title{
Design, Test and Experimental Validation of a VR Treadmill Walking Compensation Device
}

\author{
Adrian Stavar ${ }^{1}$, Laura Madalina Dascalu ${ }^{1}$, and Doru Talaba ${ }^{2}$ \\ ${ }^{1,2}$ Transilvania Univeristy of Brasov, Product Design and Robotics Department, \\ Bulevardul Eroilor, Nr. 29, Brasov, Romania \\ \{adrian.stavar, madalina.dascalu, talaba\} @unitbv.ro
}

\begin{abstract}
Virtual Reality has known exponential development in the recent years and presents important research vectors for the years to come. One major issue, unresolved yet, remains the totally immersion feeling in Virtual Environment (VE) and one of it's most important aspects still to be researched is locomotion in VE. In this direction the authors propose a bidirectional active treadmill based device adapted from an ordinary unidirectional treadmill. The device is used for researching a way to walk smoothly and in an undisturbed fashion on a very limited surface area. For controlling the treadmill's speed and direction the authors propose two simple algorithms. The implementation of the algorithms, experimental setup, tests and results are presented as well. The author's discussion including a critical perspective about the results is also reported. The final part concludes this paper with the authors' perspective and future vectors to be implemented and researched still.
\end{abstract}

Keywords: Virtual Reality, Immersion, Walking Compensation Device, Control Algorithms.

\section{Introduction}

Virtual Realty (VR) is one of the newest technological domains that had a flourishing development in the recent years. Many of the VR research directions are rapidly growing and one of the most important is represented by locomotion interfaces.

In VR, locomotion is simulated using several different types of devices, many of them evolved from physical exercise systems. There are several significant aspects that a complete locomotion device must include. One such aspect is the immersion sensation. Together with visual, acoustical and olfactory systems, a walking interface needs to create for the user a total immersion feeling.

A locomotion device used for VR purposes is a system that must provide to the user a manner that allows natural walking in the real environment and precise representation of it in the virtual environment, ensuring an adequate immersion sensation. Influences from the walking system, tracking system and other additional devices have to minimally affect the user's locomotion. Another imperative aspect of a walking simulator is safety which must be set at high standards.

In the simplest VR walking scenario, a mouse, keyboard or joystick controls displacement [1]. More complex scenarios involve tracking the user's position using 
cameras attached to a Head Mounted Display (HMD). Different approaches use a Cave Automatic Virtual Environment (CAVE) system comprised of screens used for image projection and magnetic sensors for motion detection.

Several other researchers have been conducting studies on treadmill based devices for use in VR. The first locomotion simulations were done using passive treadmills. In one such case [2] in order to move the rolling band the user uses his strength to push the band, sustained by a pair of auxiliary handlers. Because of user's effort to actuate the rolling band, the locomotion is considered to be unnatural. Subsequently, passive treadmill devices were replaced by active ones [3], [4], [5], [6], [7], [8], [9], [10]. The major advantage of active treadmill locomotion systems is given by the natural and realistic walking sensation felt by the user.

In this paper, we focus on presenting our treadmill based system along with two algorithms used for controlling its speed and sense, adapted to the user's walking style. The user's walking parameters are measured using an electromagnetic Flock of Birds tracker. Real-time capture and processing of these signals is a key feature for the proposed device.

For providing answers to our research questions, tests including the proposed algorithms were developed on eight adult persons. Test method, a short questionnaire, the obtained results and a critical discussion are presented as well.

The final part concludes this paper with the authors' perspectives and future directions still to be researched.

\section{Contribution to Sustainability}

Human locomotion inside Virtual Environments is an important concept still to be integrated and supported by novel technologies, promising to expand into most of today's domains, like medical-rehabilitation, engineering-architecture, urban planning and also into the concept of tomorrow's Future Internet - the Internet of Services.

The "bridge" that helps achieve virtual walking is represented by locomotion interfaces. By canceling the user's displacement while walking on a limited surface, an infinite new virtual space is available to use and explore. Our small dimensions system manages to reduce the main disadvantage of most active treadmill interfaces - the need for a large walking area [4], [7], [9] - and ensure proper integration in a full immersive CAVE system. To expand functionality, the two control algorithms we propose, properly manage to assist the system to support a natural user's walking sensation. The small dimensions of our proposed bidirectional treadmill system along with its developed control system make the device ideal for future immersion needs.

The goal of the presented system, algorithms and tests is to obtain preliminary data for a complete omni-directional system that we intend to develop.

The proposed system will help future development of already existing domains by opening a path to new ideas and trends for self-sustained and advanced supportive environments. We anticipate that the results presented in this paper will also contribute to VR locomotion interfaces evolution, which represent in our view a small step for sustaining future technologies' development. 


\section{Walking Compensation Device}

\subsection{The Compensation System}

In this section we present our approach for a walking compensation device, based on a classical jogging treadmill adapted for VR purposes.

The main concept of unlimited walking on a treadmill interface is based on permanently controlling the user position by trying to keep him at a reference position ("dead zone" [4]) set in the middle of the band's belt. The system always tries to keep the user in this area or very close to it were his movements and locomotion are safe and where the controller applies zero speed to the belt. Any deviation of the user's centre of mass outside the reference zone is corrected by the control system.

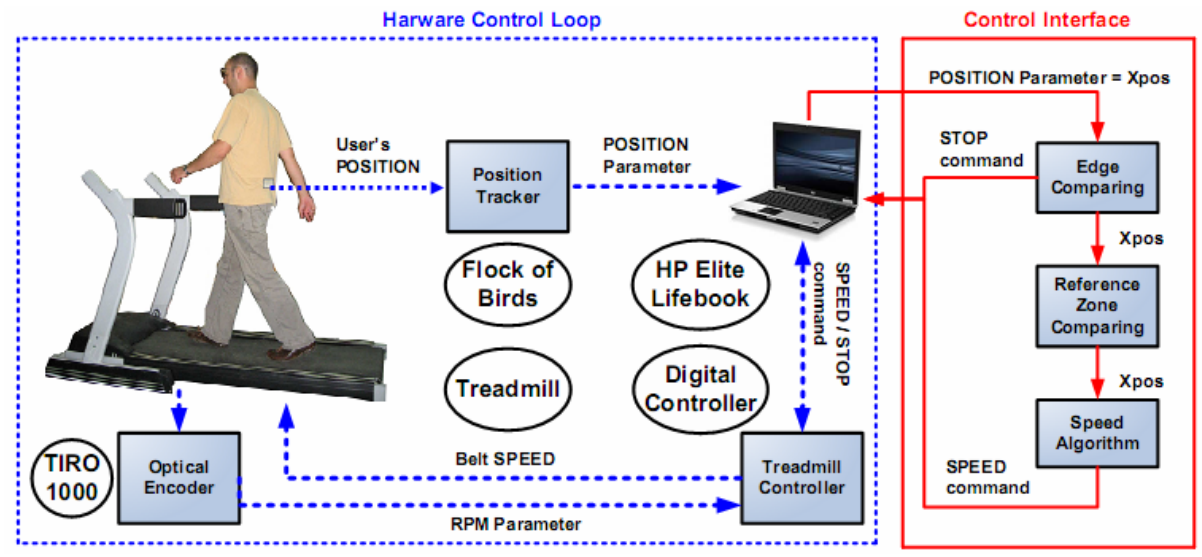

Fig. 1. System Architecture including a Control Architecture with the Hardware Control Loop (dotted line) and the Control Interface (solid line). User's position parameter (on X axis) during walking is captured with a Flock of Birds (FOB) tracker and sent to Control Interface. According to the user's position relative to reference zone ("dead zone") and edges of the treadmill's belt and following the speed control algorithm, the Control Interface sends a specific value to the Treadmill Controller. This sets the appropriate belt speed. The optical encoder closes the control loop by sending the treadmill's belt RPM signals to the controller.

Our system was implemented by adapting a classical unidirectional treadmill of $150 \mathrm{~cm}(\mathrm{~L})$ by $50 \mathrm{~cm}(\mathrm{~W})$ surface area into a bidirectional VR locomotion device (in Fig. 1, left). From the initial structure only some basic elements were reused, like treadmill structure, belt and DC motor.

The general system architecture (Fig. 1) of the adapted design includes the following subsystems:

a) HP Elite Lifebook 6930p Laptop, Core2 Duo, 2.40 GHz and 2048 MB RAM

b) Forward and reverse digital controller - controls precisely the motor's speed and position and it communicates with the computer through a serial interface (RS232).

c) Flock of Bird (FOB) magnetic motion tracker system - simultaneously uses 2 tracking sensors to detect the user's position and head orientation.

d) Optical incremental quadrature encoder module kit and a 1000 PPR encoder. 
The software application includes serial transmission configuration, Flock of Bird data transmission and position reception, parameter recording, position data and control parameters display windows. The two control algorithms were included in the control interface.

The system's safety measures include limits imposed by the algorithms to avoid the case of a user falling off the platform, kill button for stopping the system in dangerous situations and side handlers on the treadmill for stability.

\subsection{Control Algorithms}

In order for an algorithm to be adequate for walking compensation it has to be able to adaptively control the system by applying an appropriate belt speed opposite to the user's walking speed and sense. Its main role is to minimize the possibility of getting close to the treadmill's edges. In order to test our implemented system we proposed two compensation algorithms, tested in parallel for a better comparison. Our research goal was to determine if we can obtain walking compensation in a limited space area design and what is the proper method to apply.

The first algorithm is based on the idea that when the user starts walking outside the "dead zone" the system has to remotely affect his motion by minimizing inertia and friction forces. The system responds by permanently detecting and comparing the user's position on the walking belt with a set of predefined zones used for transmitting to the treadmill's controller the correct belt speed values. For each forwardbackward walking direction, the area is divided into eight speed regions, the first five of low speed increment and the last three of fast speed increment. Dividing the walking area into slow speed system response in the first half and fast speed system response in the last half was based on the idea that the user's balance is affected more in the starting moment and in the first few steps than when the speed is medium and the walking cycle becomes regular.

The second algorithm is based on the idea that the system response has to be smooth not only when the user starts walking but on the entire walking surface. The walking area was divided into 95 equal speed regions for each walking sense. The speed was increased slowly when the user position covered these regions. This slower-linear system reaction had a better influence on the walking compensation than in the first algorithm case.

The pseudo code of the 1st algorithm (Xpos is the detected user position along the $\mathrm{X}$ axis)

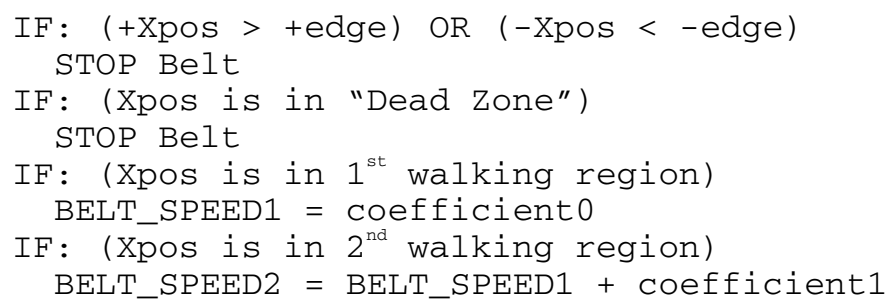




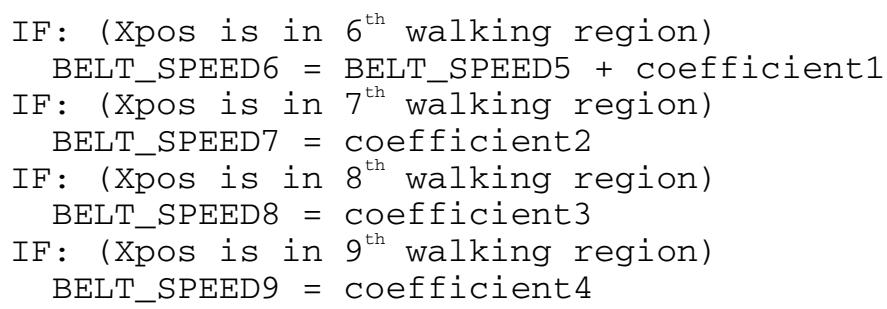

The pseudo code of the 2 nd algorithm (Xpos is the detected user position along the $\mathrm{X}$ axis)

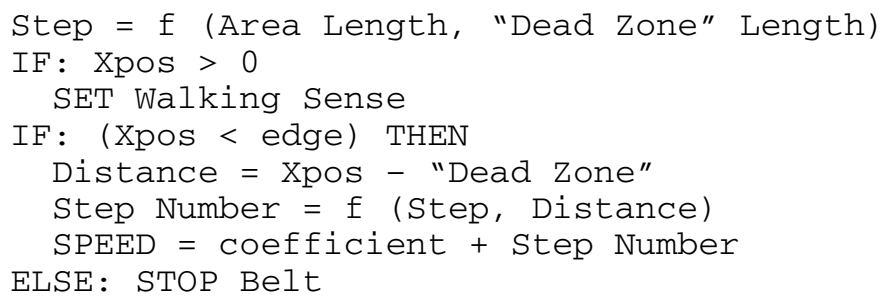

\subsection{Testing Method}

In order to validate the compensation system's functionality and to determine which of the control methods is the adequate solution for further development we conducted a testing session that included recording and comparison between walking parameters and a questionnaire based evaluation. The tests were performed without including any VR image projection system.

Eight young healthy adults participated in the test (three women and five men) with ages between $24 \div 26$ years $(25.375 \pm 0.74)$, heights between $164 \div 182 \mathrm{~cm}$ $(176.125 \pm 6.49)$ and weights between $52 \div 82 \mathrm{~kg}(67 \pm 10.32)$.

In order to measure and record the walking parameters, a FOB receiver sensor was attached on the user's lower back very close to the center of mass.

Three of the users were somewhat familiar with walking on the treadmill. The remaining five were not familiar at all. All of them were allowed to adapt with the system for 10 minutes, doing a free walk session. After the accommodation session 5 minutes resting was allowed before tests began. The two algorithms were tested sequentially without any pause in between. The test included two forward $(0-40 \mathrm{~s}$, $60-100 \mathrm{~s})$ and two backward $(40-60 \mathrm{~s}, 100-120 \mathrm{~s})$ free walking sessions. The application recorded the successive FOB receiver positions, speeds and accelerations during free walking and also the belt's speeds at every $125 \mathrm{~ms}$.

At the end of the testing session a short questionnaire (five questions) was conducted in order to obtain some prompt answers about the walking naturalness and general feelings about the walking compensation system.

\subsection{Test Results}

The average values of the users' speed, acceleration and band speed both for backward and forward algorithms are represented in Table 1. Standard errors and standard deviations are represented as well for each case. 
Table 1. Comparative tests locomotion parameters

\begin{tabular}{lllll}
\hline & & $\begin{array}{l}\text { Users' } \\
\text { Speed }\end{array}$ & $\begin{array}{l}\text { Walking } \\
\text { Uccers'eration }\end{array}$ & $\begin{array}{l}\text { Walking } \\
\text { Acceadmill's Belt }\end{array}$ \\
\hline $\mathbf{1}^{\text {st }}$ Algorithm & Mean & $0.093(\mathrm{~m} / \mathrm{s})$ & $0.521\left(\mathrm{~m} / \mathrm{s}^{2}\right)$ & $0.681(\mathrm{~m} / \mathrm{s})$ \\
Forward & Std. Error & 0.008 & 0.045 & 0.009 \\
Walking & Std. Deviation & 0.017 & 0.091 & 0.244 \\
\hline $\mathbf{1}^{\text {st }}$ Algorithm & Mean & $-0.097(\mathrm{~m} / \mathrm{s})$ & $-0.551\left(\mathrm{~m} / \mathrm{s}^{2}\right)$ & $-0.511(\mathrm{~m} / \mathrm{s})$ \\
Backward & Std. Error & 0.014 & 0.086 & 0.016 \\
Walking & Std. Deviation & 0.028 & 0.172 & 0.259 \\
\hline $\mathbf{2}^{\text {nd Algorithmm }}$ & Mean & $0.104(\mathrm{~m} / \mathrm{s})$ & $0.529\left(\mathrm{~m} / \mathrm{s}^{2}\right)$ & $0.731(\mathrm{~m} / \mathrm{s})$ \\
Forward & Std. Error & 0.010 & 0.052 & 0.008 \\
Walking & Std. Deviation & 0.020 & 0.105 & 0.216 \\
\hline $\mathbf{2}^{\text {nd } \quad \text { Algorithm }}$ Mean & $-0.110(\mathrm{~m} / \mathrm{s})$ & $-0.583\left(\mathrm{~m} / \mathrm{s}^{2}\right)$ & $-0.568(\mathrm{~m} / \mathrm{s})$ \\
Backward & Std. Error & 0.018 & 0.086 & 0.011 \\
Walking & Std. Deviation & 0.036 & 0.172 & 0.211 \\
\hline
\end{tabular}

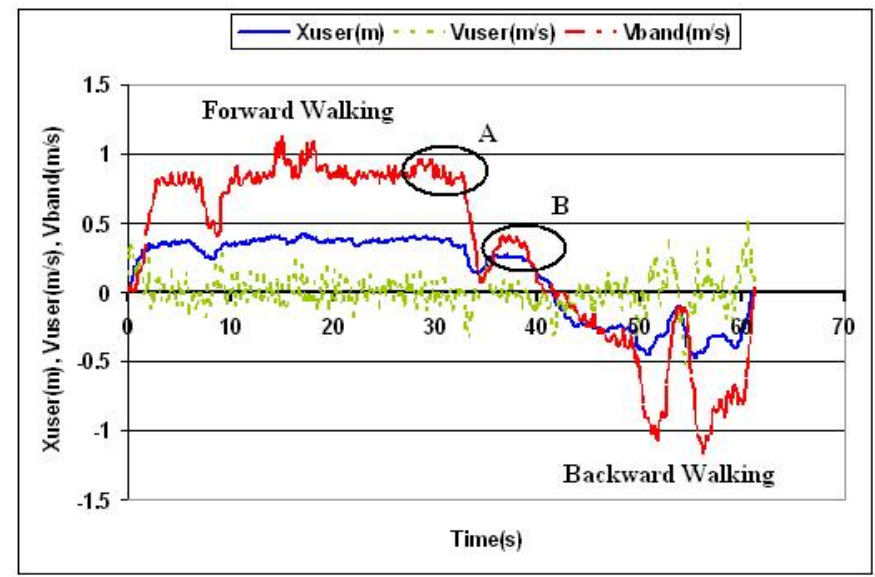

Fig. 2. A user's walking recording: $(A)$ faster compensation, $(B)$ slower compensation, user's displacement (solid line), user's speed (dotted line) and band's speed (dash-dot line)

In Fig. 2 the walking pattern of the $1^{\text {st }}$ user, including his speed and the treadmill's belt speed, is represented.

From the questionnaire responses it resulted that all users were healthy and did not suffer from any mobility disorder. The responses regarding differences between the two walking sessions were: $50 \%$ small, $37.5 \%$ average and $12.5 \%$ no differences. The first algorithm has been seen as reacting a little slower than the second and imposing a slightly insecure locomotion. For the second algorithm it was thought faster than the first in speed and reaction, more adapted and walking was more natural and comfortable. Some general issues regarding both algorithms were expressed. In both cases a small walking imbalance appears when changing walking sense (from forward to backward walking). Also the $5 \mathrm{~cm}$ "dead zone" was reported as being too narrow. $62.5 \%$ did not lose their balance, $25 \%$ lost their balance a few times in the $1^{\text {st }}$ session/algorithm, and $12.5 \%$ lost balance once in the $2^{\text {nd }}$ algorithm. Considering 
walking naturalness, $75 \%$ suggested that the second algorithm is more adapted, 12.5 $\%$ stated the first algorithm and $12.5 \%$ said that there is no difference between them.

\subsection{Test Discussion}

The results noted in Table 1 show that all the average parameters, including users' speed and acceleration as well as band's speed, are higher for the second algorithm case. Thus, we can state that the moving belt response countering the user's walking advance is better in the second algorithm. In the first algorithm walking on the first one half of the active area is softly compensated and the speed increases roughly on the last half. Because belt speed is lower in the first part the user tends to increase his speed more in order to force the system to give a better response. The system reaction is better in the second algorithm because it reacts linear and faster to the user's displacement.

Analyzing the questionnaire answers we can assert that there are no major differences between the two algorithms. Most of the users had comfortable walking sessions without losing their balance, although most of them indicated that the second algorithm is compensating better, giving them a more natural feeling.

Even though we have implemented two different walking compensation approaches with fair results, we can sustain that a proper compensation is also dependent of system hardware and especially of data transmission between the controlling application and system controller. A slower data transmission is a major drawback that can be overcome only by a fast and precise algorithm.

We can conclude that an ideal algorithm has to balance walking speed with limiting inertia and unaffecting walking. Ideally the band's speed and the user's walking speed should be almost alike. This means that the system must react very fast to any user step outside the "dead zone" area. But this affects user locomotion start. Thereby it should be a compromise between system reaction and walking disruption.

\section{Conclusion and Future Work}

Treadmill based systems for simulating locomotion remains one of the major research issues for the Virtual Reality. Creating a totally immersion feeling for the user during traveling in Virtual Environments is an important goal to be researched still.

The question, if a limited area treadmill can provide a proper walking compensation for a small to moderate unaffected and natural walking, arises. In this paper we focus on providing some answers to this question.

A classic treadmill adaptation for VR purposes and the resulting system hardware and software components are presented. Two control algorithms needed for compensating the user's locomotion are also described.

A test and questionnaire on eight young adult persons was conducted to give an opinion about the proper method of walking compensation including the developed algorithms. Test results, including a higher compensating treadmill's band speed and a higher users' walking speed, as well as users' answers of $75 \%$ indicating that the $2^{\text {nd }}$ implemented algorithm induces a more natural walking sensation, led to the conclusion that the $2^{\text {nd }}$ method was more appropriate for walking compensation. 
As a future development direction we intend to apply a fuzzy logic control algorithm in order to improve the system's performance. Also we intend to implement a rotational platform integrated in the compensation system to achieve omni-directional walking. Our intention is to research and develop a complete virtual locomotion system with application in actual and future areas of interest.

Acknowledgments. This paper is supported by the Sectoral Operational Programee Human Resources Development (SOP HDR), financed from the European Social Fund and by the Romanian Government under the contract number POSDRU/6/1.5/S/6 for the authors (1) and by the research project IREAL contract no. 97/2007, id: 132, funded by the Romanian Council for Research CNCSIS for the authors (2).

\section{References}

1. Kim, G.J.: Designing VR Systems, The Structured Approach, pp. 3-5. Springer, London (2005)

2. Patel, K.K., Vij, S.K.: Unconstrained Walking Plane to Virtual Environment for Spatial Learning by Visually Impaired. Ubiquitous Computing And Communication Journal (2010)

3. Darken, R.P., Cockayne, W.R.: The Omni-Directional Treadmill: A Locomotion Device for Virtual Worlds. In: Proc. UIST 1997, pp. 213-221 (1997)

4. Iwata, H.: Walking About Virtual Environments on an Infinite Floor. In: Proc. of the IEEE Virtual Reality (1999)

5. Iwata, H.: Art and Technology in Interface Devices. In: Proc. of the ACM Symposium on Virtual Reality Software and Technology, pp. 1-7 (2005)

6. Christensen, R., Hollerbach, J.M., Xu, Y., Meek, S.: Inertial Force Feedback for the Treadport Locomotion Interface. In: Presence: Teleoperators and Virtual Environments, vol. 9, pp. 11-14 (2000)

7. Hollerbach, J.M.: Locomotion Interfaces. In: Stanney, K. (ed.) Handbook of Virtual Environments Technology, pp. 239-254 (2002)

8. Noma, H., Miyasato, T.: A New Approach for Canceling Turning Motion in the Locomotion Interface, ATLAS. In: Proc. of AME-DSC, vol. 67, pp. 405-406 (1999)

9. Giordano, P.R., Souman, J.L., Mattone, R., De Luca, A., Ernst, M.O., Bulthoff, H.H.: The CyberWalk Platform: Humna-Machine Interaction Enabling Unconstrained Walking through VR. In: First Workshop for Young Researchers on Human-Friendly Robotics (2008)

10. De Luca, A., Mattone, R., Giordano, P.R., Bulthoff, H.H.: Control Design and Experim ental Evaluation of the 2D CyberWalk Platform. In: IEEE/RSJ IROS 2009, pp. 5051-5058 (2009) 